\section{Quality photons} from nanocrystals

Photons that are suitable for quantum communication can be emitted from solid materials.

A roadblock to building quantum information networks is the need for streams of identical photons that oscillate in sync with each other. Such streams are produced by lasers, but integrating these with electronics is difficult.

Now Mete Atatüre at the University of Cambridge, UK, and his colleagues report that they have generated such a stream from semiconducting nanocrystals, or quantum dots. These dots exhibit a phenomenon called resonance fluorescence, in which emitted photons resonate with one another, and so oscillate in sync.

Nature Commun. 4, 1600 (2013) MICROBIOLOGY

\section{Whole sequence, no culture}

The development of a culture-free method for sequencing the genomes of chlamydia-causing bacteria means that researchers can obtain genomes directly from clinical samples and skip the tricky, time-consuming process required to grow these bacteria.

Helena Seth-Smith and Nicholas Thomson at the Wellcome Trust Sanger Institute in Hinxton, UK, and their colleagues purified Chlamydia trachomatis (pictured) from genital swabs using antibody-encrusted magnetic beads that latch onto C. trachomatis cells - leaving

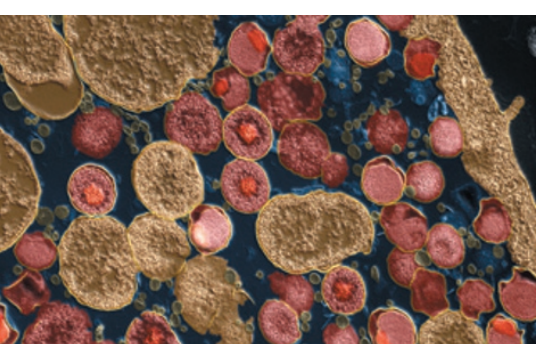

behind contaminants such as human cells and other microbes. Bacterial DNA was then copied, sequenced and assembled using bioinformatic software; this yielded five complete genome sequences from 18 samples tested.

The technique could also be used to sequence other difficult-to-culture pathogens, the authors say.

Genome Res. http://dx.doi. org/10.1101/gr.150037.112 (2013)

\section{NEUROSCIENCE}

\section{Fickle wiring in ageing brains}

The decline of cognitive ability seen in some elderly people may not result from fewer brain cells, but from a rise in capricious connections.

Vincenzo De Paola at Imperial College London, Sen Song of Tsinghua University in Beijing and their colleagues observed the cell connections that shoot signals through the brain by fitting glass windows into the craniums of old and young mice. Although the researchers expected older mice to have fewer firing points, they found more. But these connections were erratic. In older mice, the connections had high turnover rates, and wavering firing strength. The older mice also performed less well on a memory test.

The researchers suggest that mental decline seen in ageing mice is due to disorderly wiring.

Proc. Natl Acad. Sci. USA http:// dx.doi.org/10.1073/pnas. 1218731110 (2013)

\section{PARTICLE PHYSICS}

\section{Symmetry affirmed}

An unprecedented measurement of an antiproton suggests that matter and antimatter truly mirror each other.

Gerald Gabrielse at Harvard

COMMUNITY CHOICE

The most viewed papers in science

MATERIALS SCIENCE

\title{
Making films on the edge
}

\section{HIGHLY READ \\ on www.acs.org in February}

A change in the orientation of molecular sheets in layered films may open up new uses for these materials.

Thin films are usually grown by stacking atoms horizontally, like sheets in a pad of paper. But Yi Cui and his colleagues at Stanford University in California have grown layers vertically, like dominoes balanced on their edges. The researchers started with strips of molybdenum and added sulphur or selenium atoms, which diffuse down through the molybdenum, carving nanometre-sized stripes.

The molybdenum atoms are sandwiched between sulphur or selenium atoms so that the top of the structure is made up of exposed edges. These sheet-edge structures are reactive, and so might make good catalysts, the authors say. As an example, they show that a reaction to produce hydrogen gas proceeds more rapidly with a higher concentration of sheet edges.

Nano Lett. 13, 1341-1347 (2013)
University in Cambridge, Massachusetts, and his group began with a particle trap designed to measure the proton's magnetic moment - the amount of force that its magnetic spin can exert. They shipped the equipment to the world's largest particlephysics laboratory - CERN near Geneva, Switzerland - where they confined a single antiproton from the CERN beam and measured the strength of its magnetic moment. The moment of the antiproton differed from that of the proton by less than 5 parts per million, the most precise measurement ever achieved. This result is in agreement with established theory of symmetry between particles and their antimatter counterparts.

Phys. Rev. Lett. 110, 130801 (2013)

\section{APPLIED MATHS}

\section{Mechanics behind} seashell spines

Spines on seashells, which offer protection from the crushing jaws of potential predators, are often cited as an example of similar features that have evolved independently in different species. Now, their shape can be explained by the physics of shell formation.

Derek Moulton at the University of Oxford, UK, and his colleagues modelled the interactions between the hard edge of the shell and the mollusc mantle that secretes new shell material during growth. Variations in growth rates and the stiffness of new shell could account for the diversity of spine shapes found on the shells of different species in sea-snail families such as Muricidae.

A mechanical model could explain the similarities in shell spines seen across various species, the authors suggest.

Proc. Natl Acad. Sci. USA http://dx.doi.org/10.1073/ pnas.1220443110 (2013)

\section{$\rightarrow$ NATURE,COM}

For the latest research published by Naturevisit: 